\title{
EFECTO DE LAS VARIABLES CLIMÁTICAS SOBRE LA FLUCTUACIÓN DEL NIVEL FREÁTICO EN SUELOS FLUVISOLES DEL VALLE DEL RÍO CARRIZAL
}

\section{EFFECT OF VARIABLES ON CLIMATE WATER TABLE FLUCTUATION SOILS FLUVISOLS RIVER VALLEY CARRIZAL}

\author{
Veris Antonio Saldarriaga Lucas ${ }^{1 *}$, Jesús Enrique Chavarría Párraga ${ }^{2}$, Ángel Monserrate Guzman Cedeño ${ }^{1,3}$, Néstor Leopoldo \\ Tarazona Meza' ${ }^{1}$. \\ ${ }^{1}$ Escuela Superior Politécnica Agropecuaria de Manabi-Manuel Félix López, Manabí, Ecuador. \\ ${ }^{2}$ Pontificia Universidad Católica del Ecuador Sede Manabi, Manabí, Ecuador. \\ ${ }^{3}$ Universidad Laica Eloy Alfaro de Manabi, Manabi, Ecuador. \\ *Correspondencia: veris.saldarriaga@espam.edu.ec
}

Rec.: 10.12.2019. Acept.: 30.11.2020.

Publicado el 30 de diciembre de 2020

\begin{abstract}
Resumen
L a presente investigación se realizó, desde diciembre 2016 a noviembre del 2017, en el Campus Politécnico de la ESPAM MFL, en el sitio El Limón, parroquia Calceta, cantón Bolívar, con el propósito de identificar los efectos de las variables climáticas sobre la fluctuación del nivel freático en suelos fluvisoles del valle del Río Carrizal, se instalaron 16 pozos de observación en todo el campus, cada dos semanas se midió el nivel freático utilizando un flexómetro, se midió en centímetros. Las variables independientes registradas fueron: precipitación $(\mathrm{mm})$, temperaturas $\left({ }^{\circ} \mathrm{C}\right.$ máxima, mínima y ambiental), humedad relativa (\%), heliofanía $\left(\mathrm{h} . \mathrm{s}^{-1}\right)$, evaporación $(\mathrm{mm})$ y velocidad del viento $\left(\mathrm{m} . \mathrm{s}^{-1}\right)$. El análisis de datos se realizó en dos fases inicialmente: análisis descriptivo, luego para establecer relación entre las variables se utilizó el coeficiente de correlación de Pearson y finalmente un análisis de regresión múltiple, en cuanto a las variables evaluadas estadísticamente, se comprobó que el nivel freático tiene relación con la temperatura máxima, mínima, ambiental, precipitación, evaporación y heliofanía, obteniendo diferencia significativas $p<0.05$, en cuanto al análisis de regresión entre las variables predictoras y el nivel freático se obtuvieron varios modelos estadístico como alternativas para predecir el comportamiento del nivel freático, siendo el modelo ganador con un $\mathrm{R}^{2 \text { ajustado }}=0,94$, por lo tanto, se concluye el $94 \%$ del comportamiento del nivel freático se encuentra determinado por factores climáticos siendo estos: la temperatura, precipitación, evaporación y heliofanía, quienes ejercen cambios contundentes en el ascenso y descenso del mismo.
\end{abstract}

Palabras clave: Nivel freático, variables climáticas, relaciones climáticas.

\begin{abstract}
$\mathrm{T}$ his research was carried out, from December 2016 to November 2017, on the campus of the ESPAM MFL, at the Limón site, Calceta parish, Bolívar canton, with the purpose of identifying the effects of climatic variables on fluctuation from the groundwater level in fluvisoles soils of the Carrizal River Valley, 16 observation wells were installed throughout the campus, every two weeks the groundwater level was measured using a flexometer, measured in centimeters. The independent variables recorded were: precipitation $(\mathrm{mm})$, temperatures $\left({ }^{\circ} \mathrm{C}\right.$ maximum, minimum and environmental), relative humidity (\%), heliophany $\left(\mathrm{h} \mathrm{s}^{-1}\right)$, evaporation $(\mathrm{mm})$ and wind speed $\left(\mathrm{m} \mathrm{s}^{-1}\right)$. The data analysis was carried out in two phases initially: descriptive analysis, then to establish a relationship between the variables it Pearson's correlation coefficient was used and finally a multiple regression analysis, regarding the variables statistically evaluated, it was found that the level water table is related to the maximum, minimum, environmental temperature, precipitation, evaporation and heliophany, obtaining significant difference $p<0.05$, in terms of the regression analysis between the predictive variables and the water table several statistical models were obtained as alternatives to predict the behavior of the phreatic level, being the winning model with an $\mathrm{R}^{2}$ adjusted $=0.94$, therefore, $94 \%$ of the behavior of the phreatic level is concluded is determined by climatic factors being these: the temperature, precipitation, evaporation and heliophany, who exercise blunt changes in the rise and fall of himself.
\end{abstract}

Keywords: Water table, climatic variables, climatic relationships. 


\section{Introducción}

E 1 agua es uno de los recursos más importantes del mundo, debido a que su sostenibilidad cada día se vuelve un reto más difícil; el crecimiento demográfico, la contaminación y la creciente demanda han convertido al recurso hídrico en un bien escaso (Gosling \& Arnell, 2016; Delgado \& López-Vera, 2005). A su vez el clima se encuentra íntimamente relacionados con el ciclo hidrológico y sus efectos sobre los recursos hídricos, siendo el clima quien ejerce su efecto, mediante una serie de variables relevantes como: humedad ambiental, temperatura, precipitaciones (Martínez \& Patiño, 2012). En los últimos años se han producido cambios inequívocos en el clima, que se manifiestan: en aumento de temperatura mundial; mostrando conmoción no por el aumento sino por su ritmo acelerado de cambio, disminución de las precipitaciones; la observación de estas demuestran que ha generado un cambio en la cantidad, frecuencia e intensificación de los patrones hidrológicos (IPCC, 2007; Useros, 2012).

Un estudio reciente de las tendencias y cambios del clima realizado por Arroyo (2011) afirma que la temperatura continuará subiendo y la precipitación disminuyendo para la región de Sudamérica. En cuanto a los previsibles efectos que el cambio climático puede producir en los recursos hídricos y especialmente en las aguas subterráneas, Pernía \& Fornés (2009) indican que es necesario realizar una serie de investigaciones considerando nuevos escenarios producidos por el aumento generalizado de las temperaturas, disminución y mayor concentración de las precipitaciones y aumento del nivel del mar.

Por otra parte, se reconoce que los modelos matemáticos son una herramienta de la que se dispone en la actualidad, para obtener estimaciones de cambios cuantitativos (Gutiérrez \& Pons, 2006). Estos pueden ser creados con diferentes finalidades, aunque en general se resume a predicción y/o estimación del tamaño/ significación del efecto observado (Harrell, 2001). El objetivo principal de estos modelos es cuantificar la probabilidad de que ocurra el criterio de valoración dado las condiciones o factores incluidos en el modelo e idealmente reproducir estos resultados en poblaciones diferentes (Núñez, Steyerberg \& Núñez, 2011).

La modelación es una alternativa para estimar el cambio de los niveles freáticos, considerando a las condiciones climáticas variables predictoras, quienes probablemente determinan su comportamiento, considerando este contexto el propósito de la presente investigación fue: identificar las relaciones entre niveles freáticos y las variables climáticas en suelos fluvisoles del valle del Rio Carrizal.

\section{Materiales y métodos}

I a investigación se realizó en el Campus Politécnico de la Escuela Superior Politécnica Agropecuaria de Manabí "Manuel Félix López", situada a 15,5 m. sobre el nivel del mar, en el sitio El Limón, parroquia Calceta, cantón Bolívar, provincia de Manabí, a 0049'27.9" de Latitud Sur y $80^{\circ} 10^{\prime} 47.2^{\prime \prime}$ de Longitud Oeste. La investigación fue de tipo no experimental, se utilizó métodos exploratorio y descriptivo, la duración fue de 12 meses entre diciembre 2016 a noviembre 2017.

La metodología que se utilizó para la recolección de datos del nivel freático fue mediante el uso de instrumentos manuales, y consistió en extender el flexómetro a través del pozo de observación, desde la superficie del suelo hasta encontrar límite del agua subterránea o zona saturada, a este espacio se le denominó como profundidad del nivel freático, el nivel freático se lo consideró como la diferencia entre el valor base de inicio de profundidad y el valor obtenido en la fecha correspondiente de toma, este procedimiento se lo realizó cada dos semanas en la época de lluvia (entre enero a junio) y época seca (julio a diciembre), el número total de pozos de observación fueron 16 distribuidos a través de todo el Campus en una superficie de 114 ha, los datos obtenidos se los almacenó en la base de datos conjuntamente con las variables climáticas: precipitación, temperatura, humedad relativa, evaporación, heliofanía y velocidad del viento, obtenidos de la estación meteorológica disponible en el Campus Politécnico de la ESPAM MFL.

En la investigación se aplicó estadística descriptiva para observar el comportamiento de las variables en estudio, luego se realizó un análisis de correlación y regresión múltiple entre las variables climáticas y el nivel freático, con el fin de establecer el comportamiento del nivel freático frente a las variables climáticas, para las variables que no cumplían con el supuesto de homogeneidad se les realizó una transformación de datos mediante. Finalmente se realizó los freatimétricos, los cuales demuestran la variación temporal del nivel freático con relación a la precipitación y temperatura.

\section{Resultados y discusión}

$\mathrm{D}_{1}^{\mathrm{e}}$ acuerdo a los resultados presentados en el cuadro para el periodo de diciembre 2016 -noviembre 2017 la humedad relativa promedio de la zona fue de $83,25 \%$. En el área de estudio se registró valores de promedio anual de temperatura máxima y mínima de 30,84 y $22,06^{\circ} \mathrm{C}$ respectivamente, obteniendo un rango de oscilación de la temperatura de $8,78^{\circ} \mathrm{C}$, esta variación se debe a la zona de ubicación geográfica y por las épocas climáticas, ya que éstas determinan las condiciones 
Cuadro 1. Principales estadígrafos de las variables climáticas evaluadas al $95.0 \%$ de probabilidad de ocurrencia.

\begin{tabular}{lccccc}
\hline \multicolumn{1}{c}{ Variables } & Media & $\begin{array}{c}\text { Error } \\
\text { típico }\end{array}$ & $\begin{array}{c}\text { Desviación } \\
\text { estándar }\end{array}$ & Curtosis & $\begin{array}{c}\text { Coeficiente } \\
\text { de } \\
\text { asimetría }\end{array}$ \\
\hline Humedad relativa (HR) \% & 83,25 & 0,65 & 2,26 & 5,64 & $-2,01$ \\
Temperatura máxima (TMAX) ${ }^{\circ} \mathrm{C}$ & 30,84 & 0,33 & 1,15 & $-1,60$ & $<0,01$ \\
Temperatura mínima (TMIN) ${ }^{\circ} \mathrm{C}$ & 22,06 & 0,22 & 0,78 & $-0,24$ & $-0,33$ \\
Temperatura ambiente (TA) ${ }^{\circ} \mathrm{C}$ & 25,96 & 0,29 & 0,99 & -1.78 & $-0,02$ \\
Evaporación (E) mm & 10,00 & 4,04 & 14,01 & $-1,65$ & $-0,36$ \\
Precipitación (PP) mm & 110,07 & 43,70 & 151,37 & $-0,50$ & 1,14 \\
Horas sol (HS) H sol mes ${ }^{-1}$ & 94,13 & 8,47 & 29,33 & $-1,06$ & 0,33 \\
Velocidad del viento (VV) $\mathrm{m} \mathrm{s}^{-1}$ & 1,70 & 0,04 & 0,13 & $-1,56$ & 0,63 \\
Nivel freático (NF) cm & 74,21 & 17,53 & 60,74 & $-1,11$ & 0,6 \\
Profundidad del nivel freático (PNF) cm & 209,79 & 17,53 & 60,74 & $-1,11$ & $-0,56$ \\
\hline
\end{tabular}

climáticas. En diversos estudios realizados por Promis, Caldentey \& Ibarra (2010)the air temperature ( $\mathrm{T}$ a y Duval \& Campo (2017) indican, que las variables meteorológicas medias, se ajustan a las características propias de un punto geográfico o área reducida lo cual se representa como una modificación local del clima de la región, efecto conocido como microclima, además esto coincide con Oñate \& Valdivieso (2012) quienes publican que en el Ecuador las zonas costeras presentan características propias de climas tropicales de acuerdo con la clasificación de Koppen, determinado por la influencia del Océano Pacífico.

En la zona de valle del Rio Carrizal existen dos épocas bien diferenciadas que son: seca y lluviosa, en el cuadro 2 se evidencia las variaciones de los parámetros climáticos y nivel freático los cuales se comportan de manera distintas de acuerdo a la época del evento. En la época de lluvia se registró una precipitación de 1295,1 $\mathrm{mm}$ correspondiente al $(98,05 \%)$ de la precipitación total mientras que en la seca $25,7 \mathrm{~mm}$. La temperatura máxima corresponde a la época de lluvia alcanzando un valor de $32,3^{\circ} \mathrm{C}$, mientas que la temperatura mínima corresponde a la época de sequía llegando a temperaturas de $20,5^{\circ} \mathrm{C}$. Resultados que coinciden con Chavarría et al. (2020), los cuales manifiestan que una zona típica de la costa ecuatoriana en la época lluviosa posee una temperatura máxima que fluctúa entre $31,1^{\circ} \mathrm{C}$ y 35,3 ${ }^{\circ} \mathrm{C}$. Esto nos determina claramente la temperatura promedio del ambiente tanto en época de lluvia o de sequía registrando 26,8 y $25,2^{\circ} \mathrm{C}$ respectivamente; con un rango de $1,6^{\circ} \mathrm{C}$ entre ambas épocas y estableciendo que en época de lluvia hay un ligero aumento de la temperatura con respecto a la época seca. En cuanto a las horas sol se registra que en la época de lluvia hay mayores valores de horas sol mes en comparación a la época seca. Por otro lado, el comportamiento del nivel freático en época de lluvia se encuentra un promedio de $124,5 \mathrm{~cm}$, mientras que en época seca apenas solo 23,9 $\mathrm{cm}$ de nivel freático, lo que claramente indica que la profundidad del nivel freático en época lluviosa y seca se encuentra $159,5 \mathrm{~cm}$ y $260,1 \mathrm{~cm}$, con respecto a la superficie del suelo.

Cuadro 2. Análisis descriptivo por épocas.

\begin{tabular}{cccc}
\hline \multirow{2}{*}{ Variables } & \multicolumn{2}{c}{ Épocas } & \multirow{2}{*}{ Total } \\
\cline { 2 - 3 } & Lluviosa & Seca & \\
\hline $\begin{array}{c}\text { Suma de precipitación } \\
\text { mm }\end{array}$ & 1295,1 & 25,7 & 1320,8 \\
$\begin{array}{c}\text { Máx. de Temperatura } \\
\text { máxima }{ }^{\circ} \mathrm{C}\end{array}$ & 32,3 & 31,4 & 32,3 \\
$\begin{array}{c}\text { Mín. de Temperatura } \\
\text { mínima }{ }^{\circ} \mathrm{C}\end{array}$ & 22,0 & 20,5 & 20,5 \\
$\begin{array}{c}\text { Promedio de Temperatura } \\
\text { ambiente }{ }^{\circ} \mathrm{C}\end{array}$ & 26,8 & 25,2 & 26,0 \\
$\begin{array}{c}\text { Promedio de Evaporación } \\
\quad \text { mm }\end{array}$ & 95,8 & 110,1 & 103,0 \\
$\begin{array}{c}\text { Promedio de Horas sol } \\
\text { h sol mes }{ }^{-1}\end{array}$ & 111,1 & 77,2 & 94,1 \\
$\begin{array}{c}\text { Promedio de Vientos m s }{ }^{-1} \\
\quad 1,8\end{array}$ & 1,6 & 1,7 \\
$\begin{array}{c}\text { Promedio de Nivel } \\
\text { freático cm }\end{array}$ & 124,5 & 23,9 & 74,2 \\
$\begin{array}{c}\text { Promedio de Profundidad } \\
\text { de Nivel freático cm }\end{array}$ & 159,5 & 260,1 & 209,8 \\
\hline
\end{tabular}

Como parte fundamental de la investigación, se consideró las correlaciones parciales de las variables predictoras con la independiente. Lo cual el nivel freático presentó mayor relación con la precipitación PP $(r=$ 0.86; $\boldsymbol{p}=<\mathbf{0 . 0 1}$ ), lo cual concuerda con Gyoo-Bum, et al. (2006) quienes reportan coeficientes de correlación mayores de 0,737 entre la precipitación estandarizada 
y el nivel de agua subterránea, además indican que la precipitación estandarizada ayuda a predecir valores luego del evento. La profundidad del nivel freático es inversamente proporcional al nivel freático, quien reporta un coeficiente de correlación PP $(\boldsymbol{r}=\mathbf{- 0 . 8 6}$; $\boldsymbol{p}=$ $<\mathbf{0 . 0 1}$ ), lo que coincide con el estudio de Coras (2006) quien obtuvo coeficiente de correlación similar entre la precipitación y la profundidad del nivel freático con $-0,61$. En cuanto a la temperatura mínima, ambiental y máxima, se obtuvo coeficientes de $(\boldsymbol{r}=\mathbf{0 . 8 7 4 , 0 . 8 1 8}$ $y$ 0.764; $p=<0.01)$ respectivamente, evidenciando la relación entre la temperatura y el nivel freático, la temperatura ambiental presenta correlación con TMIN ${ }^{\circ} \mathrm{C}$ y $\mathrm{TMAX}{ }^{\circ} \mathrm{C}$ de modo que la temperatura ambiental siendo el promedio entre máxima y mínima, es una regresora clave para creación del modelo de regresión.

La evaporación muestra una correlación significativa con el nivel freático con un EVA $(\boldsymbol{r}=\mathbf{- 0 . 5 8} \boldsymbol{p} \boldsymbol{p}=<\mathbf{0 . 0 5})$, es claro que hay correlación inversa entre más evaporación exista hay una disminución de nivel freático, esto concuerda con Vidal (2009), quien reporta indicies de correlación de $46 \%$ para un ajuste de curva lineal. La cantidad de horas sol muestra una correlación positiva, es decir un aumento de horas sol determinará un aumento del nivel freático, HS $(\boldsymbol{r}=\mathbf{0 . 7 4} ; \boldsymbol{p}=<\mathbf{0 . 0 1})$. En cuanto a esto se indica que las variables climáticas precipitación, temperatura media o ambiental, evaporación y horas sol son variables climáticas determinantes para el ascenso y descenso del nivel freático, es decir una variación de estas indican un cambio evidente en el nivel freático.

Además, existe una correlación entre las variables climáticas, la precipitación en función de la temperatura, es decir que a medida que hay un aumento de temperatura hay mayor probabilidad de presentar valores más altos de precipitaciones (cuadro 3), lo que coincide con investigaciones realizada por Alzate et al. (2015); Serrano et al. (2017) quienes indican que la precipitación está influenciada por las temperaturas ambientales; sin embargo, Alzate et al. (2015) sugieren que se debe realizar un analisis más profundo para llegar a conocer su efecto en tiempo- espacio y dar dominios de predicción.

El análisis de regresión múltiple permitió explicar con exactitud el grado de correlación entre las variables en estudio, siendo significativa $p<0.05$ la influencia de las variables predictoras: precipitaciones, temperaturas, evaporación y horas sol, sobre el nivel freático, obteniendo así cuatro modelos de regresión, estableciendo al modelo ganador, al que obtuvo un $\mathrm{R}^{2}$ ajustado más alto y un AIC más bajo, siendo el modelo

Cuadro 3. Matriz de correlación lineal entre las variables de estudio.

\begin{tabular}{|c|c|c|c|c|c|c|c|c|c|c|}
\hline \multicolumn{11}{|c|}{ CORRELACIONES PEARSON } \\
\hline Variables & HR \% & $\begin{array}{c}\text { TMAX } \\
{ }^{\circ} \mathbf{C} \\
\end{array}$ & $\begin{array}{c}\text { TMIN } \\
{ }^{\circ} \mathbf{C} \\
\end{array}$ & $\begin{array}{c}\text { TMED } \\
{ }^{\circ} \mathrm{C} \\
\end{array}$ & $\begin{array}{l}\text { EVA } \\
\text { mm }\end{array}$ & $\begin{array}{c}\text { PP } \\
\text { mm }\end{array}$ & $\begin{array}{c}\text { HS sol } \\
\text { mes }^{-1}\end{array}$ & $\begin{array}{l}\mathbf{V V} \\
\mathrm{m} \mathrm{s}^{-1}\end{array}$ & $\begin{array}{l}\text { NF } \\
\text { cm }\end{array}$ & $\begin{array}{l}\text { PNF } \\
\text { cm }\end{array}$ \\
\hline$\underset{\%}{H R}$ & 1 & & & & & & & & & \\
\hline$\underset{{ }^{\circ} \mathbf{C}}{\mathbf{T M A X}}$ & $-0,123$ & 1 & & & & & & & & \\
\hline$\underset{{ }^{\circ} \mathbf{C}}{\text { TMIN }}$ & 0,187 & $0,864^{* *}$ & 1 & & & & & & & \\
\hline$\underset{{ }^{\circ} \mathbf{C}}{\text { TMED }}$ & $-0,064$ & $0,961^{* *}$ & $0,918^{* *}$ & 1 & & & & & & \\
\hline $\begin{array}{l}\text { EVA } \\
\text { mm }\end{array}$ & $-0,46$ & $-0,069$ & $-0,361$ & $-0,233$ & 1 & & & & & \\
\hline $\begin{array}{c}\text { PP } \\
\mathbf{m m}\end{array}$ & 0,143 & $0,704^{*}$ & $0,733^{* *}$ & $0,699^{*}$ & $-0,546$ & 1 & & & & \\
\hline $\begin{array}{c}\text { HS sol } \\
\text { mes }^{-1}\end{array}$ & 0,024 & $0,904^{* *}$ & $0,730^{* *}$ & $0,818^{* *}$ & 0,076 & $0,607^{*}$ & 1 & & & \\
\hline $\begin{array}{l}\mathbf{V V} \\
\mathbf{m ~ s}^{-1}\end{array}$ & $-0,251$ & $0,752^{* *}$ & $0,585^{*}$ & $0,737^{* *}$ & $-0,046$ & 0,507 & $0,680^{*}$ & 1 & & \\
\hline $\begin{array}{l}\text { NF } \\
\mathrm{cm}\end{array}$ & 0,332 & $0,764^{* *}$ & $0,874^{* *}$ & $\mathbf{0 , 8 1 8 ^ { * * }}$ & $-0,580^{*}$ & $\mathbf{0 , 8 6 0} 0^{* *}$ & $0,738^{* *}$ & 0,498 & 1 & \\
\hline $\begin{array}{l}\text { PNF } \\
\text { cm }\end{array}$ & $-0,331$ & $-0, .64^{* *}$ & $-0,874^{* *}$ & $-0,819^{* *}$ & $0,580^{*}$ & $-0,860^{* *}$ & $-0,738^{* *}$ & $-0,498$ & $-1,000^{* *}$ & 1 \\
\hline
\end{tabular}


Cuadro 4. Análisis y selección de modelos de regresión múltiple.

\begin{tabular}{|c|c|c|c|c|}
\hline \multirow{2}{*}{$\mathbf{N}^{\mathbf{o}}$} & \multicolumn{4}{|c|}{ Selección de modelos regresión múltiple } \\
\hline & 1 & 2 & 3 & 4 \\
\hline $\begin{array}{l}\text { Numero de } \\
\text { Regresoras }\end{array}$ & 2 & 3 & 3 & 4 \\
\hline CMError & 216,34 & 223,17 & 229,83 & 253,04 \\
\hline $\mathbf{R}^{2}$ & 0,95 & 0,96 & 0,95 & 0,96 \\
\hline $\mathbf{R}^{2}$ Ajustado & 0,94 & 0,94 & 0,94 & 0,93 \\
\hline p-valor & $<0.0001$ & $<0.0001$ & $<0.0001$ & 0.0001 \\
\hline Modelo & $\begin{array}{c}\mathbf{N F}=\mathbf{2 0 6 . 7 5} \\
\mathbf{- 2 . 7 8 *} \\
\text { Evaporación } \\
+\mathbf{1 . 6 3 *} \text { Horas } \\
\text { sol } / \text { mes }\end{array}$ & $\begin{array}{c}\mathbf{N F}=\mathbf{1 8 6 . 4 9}+ \\
\mathbf{7 . 6 8} * \text { Precipitación }- \\
\mathbf{2 . 5 1} * \text { Evaporación }+ \\
\mathbf{1 . 4 4} * \text { Horas sol } / \text { mes }\end{array}$ & $\begin{array}{c}\mathbf{N F}=\mathbf{4 3 . 4 8}+ \\
\mathbf{6 . 4 2} * \text { Tmedia }- \\
\mathbf{2 . 6 4}{ }^{*} \text { Evaporación }+ \\
\mathbf{1 . 4 5} * \text { Horas sol/mes }\end{array}$ & $\begin{array}{c}\mathbf{N F}=\mathbf{1 1 8 . 5 8}+ \\
\mathbf{2 . 8 3} * \text { Tmedia }+ \\
\mathbf{6 . 0 8} * \text { Precipitación }- \\
\mathbf{2 . 5 1} * \text { Evaporación }+ \\
\mathbf{1 . 4} * \text { Horas sol/mes }\end{array}$ \\
\hline AIC & 103,12 & 104,08 & 104,44 & 105,99 \\
\hline$\Delta \mathbf{i}$ & 0 & 0,96 & 1,32 & 2,87 \\
\hline$W_{i}^{*} 100$ & 100,00 & 92,84 & 91,83 & 91,81 \\
\hline
\end{tabular}

ganador $\mathbf{N F}=\mathbf{2 0 6 . 7 5}-\mathbf{2 . 7 8 *}$ Evaporación $+\mathbf{1 . 6 3} *$ Horas sol/mes, $\mathrm{R}^{2}$ ajustado=0.94; sin embargo se consideran otras alternativas de modelo tal como lo mencionan Guzmán-Castellanos, Morales-Bojórquez \& Balart (2014) que dentro de un numero de modelos candidatos se estima las diferencias de Akaike definidas como $\Delta \mathbf{i}$, estableciendo que $\Delta \mathbf{i}<2$ tiene una alta evidencia como funciones alternativas, es así que en el cuadro 4 se encuentra los modelos alternativos. En cuanto a la evidencia científica de cada una de las variables que han sido consideradas en los modelos, se sabe que la evaporación se produce por efecto de la radicación solar, el viento y las diferencias de tensión de vapor entre el agua y la atmosfera, de manera que la acción de estas calienta las capas superiores de agua, además parte del calor calienta capas profundas ocurriendo un almacenaje de calor (Vidal, 2009), sin embargo el mismo autor indica que hay otros factores climáticos adicionales, los cuales determinan el proceso de la profundidad del nivel freático.

La temperatura hace sentir su efecto sobre los niveles freáticos a través de la variación de la presión atmosférica, siendo así que una disminución de la temperatura implica un aumento de la presión atmosférica y una disminución en su capacidad para absorber humedad (Maderey Rascón \& Jiménez Román, 2001). Para complementar, se sabe que el agua sub superficial se divide en presiones de poros positivas o superiores y las negativas o inferiores a la presión atmosférica, siendo el nivel freático la linea divisoria entre estas presiones, expresando que la presión en este límite es igual a la presión atmosférica (Suarez, 2009).
En cuanto a la precipitación hay evidencia que el agua que atraviesa el límite inferior de la zona no saturada, mediante la infiltración a través del perfil de suelo se le considera una recarga. Sophocleous (2002) menciona que esta recarga produce un aumento o ascenso del nivel freático, lo que evidentemente coincide con la aplicación del modelo de regresión lineal.

Las figuras 1a, 1b, 1c, 1d, muestran el ajuste independiente de las variables climáticas en correspondencia con el nivel freático, la tendencia de datos muestra un ajuste de modelo lineal positivo para las variables temperatura media, horas sol y precipitación mientras que, para evaporación es de forma negativa, además los datos reales tienen un ajuste muy significativo con los pronósticos, lo que hace al modelo confiable por su minimización del error y por ende su predicción es más aceptable.

La fluctuación de nivel freático ascenso o descenso del mismo se debe a la variación de las variables climáticas: evaporación, horas sol, precipitación y temperatura ambiental siendo estas dos últimas las más determinantes de acuerdo a la explicación estadística. Siendo así, que el ascenso del nivel se debe a una acumulación de precipitación durante un periodo y espacio determinado sobre la capa superficial del agua subterránea. Asimismo, la temperatura ejerce su efecto sobre el nivel freático, ya que a temperaturas más altas se encuentran niveles freático más cercanos a la superficie del suelo. Investigación reciente de Pernía \& Fornés (2009) indica que el aumento de la temperatura tiene importantes consecuencias en las aguas subterráneas próximas a la superficie del terreno. 


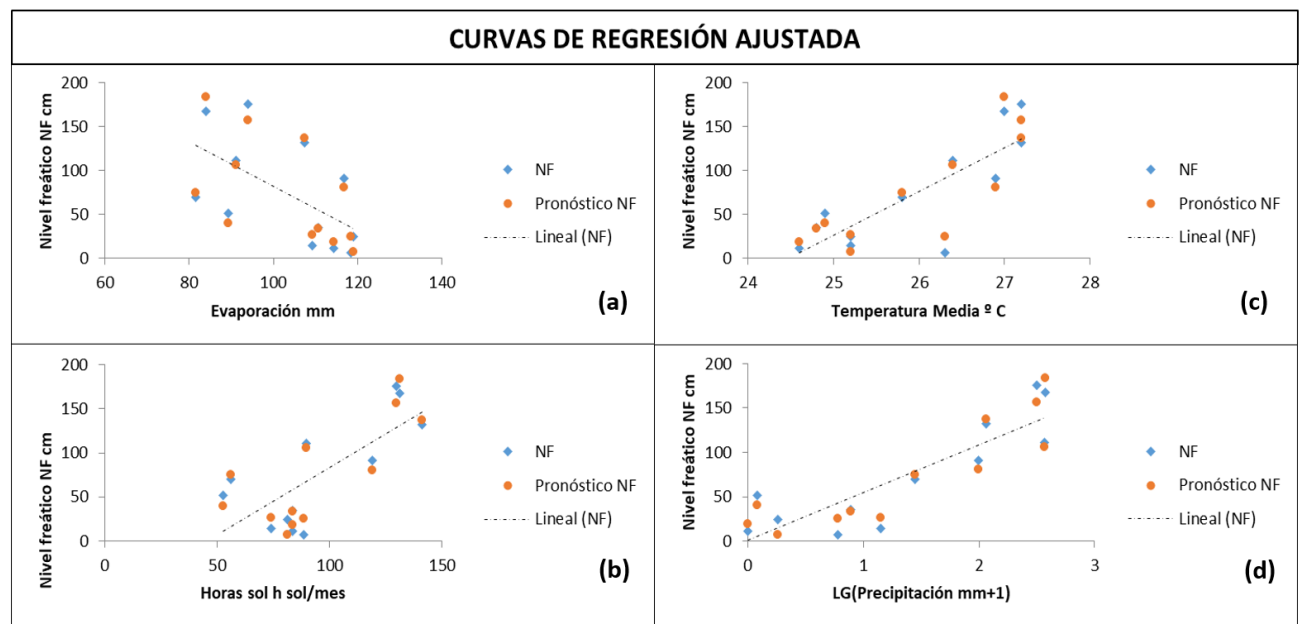

Figura 1. a. Curva de regresión ajustada de la variable climática Evaporación. b. Variable climática horas sol. c. Variable climática temperatura media. d. Variable climática precipitación.

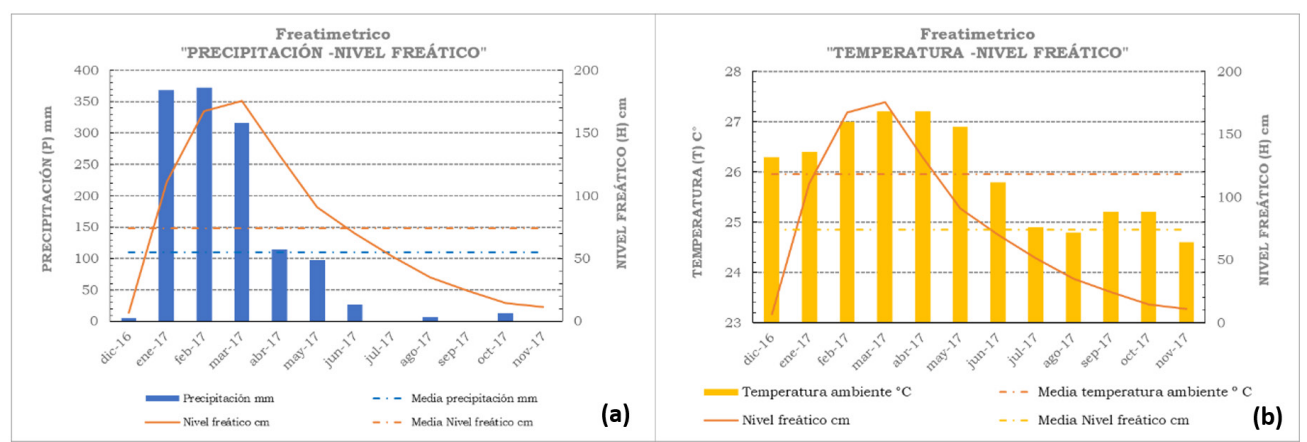

Figura 2. a. Freatimétrico anual periodo diciembre 2016-noviembre 2017 "Precipitación; Nivel freático" Espam MFL. b. Freatimétrico anual periodo diciembre 2016-noviembre 2017 "Temperatura; Nivel freático" Espam MFL.

En las figuras 2a, 2b, se observa el comportamiento anual de los niveles freático, precipitaciones $\mathrm{y}$ temperaturas climáticas, además se evidencian los ascensos y descensos de los niveles freático en función de las variables climáticas, encontrando un ascenso en meses de diciembre-marzo y un descenso en los meses de abril-noviembre, este comportamiento se debe a las épocas climáticas de las zonas, encontrando de inicio la época lluviosa por los meses de enero-junio luego la seca por los meses de julio-diciembre. Esto coincide con las publicación de Rosales, (2001) quien define que debido a la ubicación geográfica del Ecuador, no permite la presencia de las cuatro estaciones climáticas, por lo que se consideran exclusivamente las épocas secas y lluviosas, esta última iniciando en el mes de diciembre y se prolonga hasta el mes de mayo con valores máximo de la distribución en los meses de marzo o abril.

\section{Conclusiones}

$\mathrm{E}$ estudio realizado entre el nivel freático y las variables climáticas en los suelos Fluvisoles del valle del Río Carrizal permitió establecer sus interacciones.

El nivel freático y las variables climáticas, muestran un contraste en su comportamiento durante el tiempo establecido, evidenciando dos épocas claves "seca y lluviosa".

El nivel freático tiene una relación significativa con las variables climáticas: Temperatura máxima, mínima, ambiental, precipitación, evaporación y heliofanía, siendo la temperatura ambiental, precipitación, evaporación y horas sol variables contundentes en la fluctuación del nivel freático.

La evaporación, las horas sol, precipitación y temperatura como variables regresoras son de suma importancia para los modelos ya que se ajustan muy bien a las distintas alternativas de modelo de regresión múltiple siendo el mejor modelo .

Los resultados obtenidos son propios de un espaciotiempo específico, es conveniente repetir la investigación durante más tiempo y en distintas localidades, para consolidar resultados y establecer dominios predictivos. 


\section{Literatura citada}

Alzate, Diego; Rojas, Edwin; Mosquera, Jemay; Jacipt, R. (2015). Cambio climático y variabilidad climática para el periodo 1981-2010 en las cuencas de los ríos Zulia y Pamplonita, Norte De Santander - Colombia. Revista Luna Azul, (40), 127-153. https://doi.org/10.17151/ luaz.2015.40.10

Arroyo, J. (2011). Tendencias y cambio del clima del valle del Mantaro mediante los análisis de índices de Precipitación Efectiva y Temperatura Eficiente. Apuntes de Ciencia \& Sociedad, 01(01), 45-54. https://doi.org/10.18259/ acs.2011009

Chavarría. J., Ugando. M., Sabando. A., Muños. J, Bravo. R. \& Villalón, A. (2020). Necesidades hídricas del fríjol caupí (Vigna unguiculata (L.) Walp.). calculadas con el coeficiente de cultivo utilizando lisímetro de drenaje. Cien. Agri. 17(3), 111-121. https://doi. org/10.19053/01228420.v17.n3.2020.11746

Coras, P. (2006). Elemetos climáticos y su relación con la fluctuación del nivel freático. Terra Latinoamericana, 24, 375-382.

Delgado, C. D., \& López-Vera, M. V. E. A. F. (2005). Recursos Hídricos. Conceptos básicos y estudios de caso en Iberoamérica. In Recursos Hídricos. Conceptos básicos y estudio de caso en iberoamerica.

Duval, V., \& Campo, A. (2017). Variaciones microclimáticas en el interior y exterior del bosque de caldén (Prosopis caldenia), Argentina. Cuadernos de Geografia: Revista Colombiana de Geografia, 26(1), 37-49. https://doi. org/10.15446/rcdg.v26n1.42372

Gosling, S. N., \& Arnell, N. W. (2016). A global assessment of the impact of climate change on water scarcity. Climatic Change, 134(3), 371-385. https://doi.org/10.1007/ s10584-013-0853-x

Gutiérrez, J., \& Pons, M. (2006). Modelización numérica del cambio climático: bases científicas, incertidumbres y proyecciones para la Península Ibérica. Cuaternario y Geomorfología: Revista de La Sociedad Española de Geomorfología y Asociación Española Para El Estudio Del Cuaternario, 20(3), 15-28.

Guzmán-Castellanos, A. B., Morales-Bojórquez, E., \& Balart, E. F. (2014). Estimación del crecimiento individual en elasmobranquios: La inferencia con modelos múltiples. Hidrobiologica, 24(2), 137-150.

Gyoo-Bum, Kim; Han-Heum, Yun; Dae-Ho, K. (2006). Relationship Between standardized precipitation Index and Groundwater levels a proposal for establishment of drought index wells. 2006.pdf. Revista de La Sociedad Coreana de Medio Ambiente Del Suelo y Aguas Subterráneas, 11, 31-42.

Harrell, F. (2001). Regression modeling strategies: with applications to linear models, logistic regression, and survival analysis. (Springer-V). New York.
IPCC, I. P. on C. C. (2007). [Climate change 2007]: The physical science basis : summary for policymakers and technical summary and frequently asked questions; part of the Working Group I contribution to the Fourth Assessment Report of the Intergovernmental Panel on Climate Change. Proceedings of the Alpine Snow Workshop, Munich, October 5-6, Germany | HeBIS-Verbundkatalog, 8, 142. Retrieved from http:// bluemarble.nasa.gov.

Maderey Rascón, L. E., \& Jiménez Román, A. (2001). Alteración del ciclo hidrológico en la parte baja de la cuenca alta del río Lerma por la transferencia de agua a la Ciudad de México. Investigaciones Geográficas, 1(45). https://doi.org/10.14350/rig.59143

Martínez, P., \& Patiño, C. (2012). Efecto Del Cambio Climatico En La Disponibilidad De Recursos Hidricos. Tecnologia y Ciencias Del Agua, 3, 5-20.

Núñez, E., Steyerberg, E. W., \& Núñez, J. (2011). Estrategias para la elaboración de modelos estadísticos de regresión. Revista Espanola de Cardiologia, 64(6), 501-507. https://doi.org/10.1016/j.recesp.2011.01.019

Oñate Valdivieso, F. R. (2012). Proyecto: "Generación De Geoinformación Para La Gestión Del Territorio a Nivel Nacional Escala 1: 25 000.” In Memoria Técnica. https:// doi.org/10.1017/CBO9781107415324.004

Pernía, J., \& Fornés, J. (2009). Cambio climático y agua subterráea. Environmental and Engineering Geoscience, 17(2), 172-178. https://doi.org/10.2113/ gseegeosci.15.3.211

Promis, A., Caldentey, J., \& Ibarra, M. (2010). Microclima en el interior de un bosque de Nothofagus pumilio y el efecto de una corta de regeneración Microclimate within a Nothofagus pumilio forest and the effects of a regeneration felling. Bosque, 31(2), 129-139. Retrieved from https://scielo.conicyt.cl/pdf/bosque/v31n2/art06. pdf

Rosales, G. O. (2001). Evidencia de cambio climático y variabilidad climática en el Ecuador.

Serrano, S., Zuleta, D., Moscoso, V., Jácome, P., Palacios, E., \& Villacís, M. (2017). Análisis estadístico de datos meteorológicos mensuales y diarios para la determinación de variabilidad climática y cambio climático en el Distrito Metropolitano de Quito. La Granja, 16(2), 23. https://doi.org/10.17163/lgr.n16.2012.03

Sophocleous, M. (2002). Interactions between groundwater and surface water: The state of the science. Hydrogeology Journal, 10(1), 52-67. ttps://doi.org/10.1007/s10040001-0170-8

Suarez, J. (2009). Deslizamiento: Analisi geotecnico (Universida). España.

Useros, L. J. (2012). El Cambio Climático: Sus Causas Y Efectos Medioambientales. Real Academia de Medicina y Cirugía de Valladolid, 50, 71-98.

Vidal, J. J. (2009). Evaporación desde napas freáticas 
Saldarriaga et al., 2020

someras en cuencas endorreicas del altiplano chileno. Retrieved from. http://200.55.208.189/xmlui/ handle/123456789/1366 